\title{
ÉTAT DES POPULATIONS DE L'UMBRE PYGMÉE UMBRA PYGMAEA (DE KAY, 1842) DANS LE DÉPARTEMENT DE LA MARNE.
}

\author{
F. GUIDOU (1), P. KEITH (2)*
}
(1) Brigade CSP Marne, 3 rue Notre Dame, F-51300 CHATELRAOULD, France.
(2) Laboratoire d'ichtyologie, Muséum National d'Histoire Naturelle, 43 rue Cuvier, F-75231 PARIS Cedex 05, France.
* auteur correspondant

\section{RÉSUMÉ}

De nombreux individus de l'espèce Umbra pygmaea (De Kay, 1842), famille des Umbridae, ont été capturés en novembre 2000 par pêche électrique dans le département de la Marne près de Givry en Argonne, dans l'étang de Braux-forêt. Cette espèce avait été introduite en France au début du siècle.

\section{COMMUNITIES OF UMBRA PYGMAEA (DE KAY, 1842) IN FRANCE IN THE MARNE BASIN.}

\section{SUMMARY}

Numerous specimens of Umbra pygmaea (De Kay, 1842), Umbridae family, were captured in November 2000 by electric fishing in a French area, the department of the Marne near Givry en Argonne, in the pond of Braux-forêt. This species had been introduced in France at the beginning of the century.

L'umbre pygmée est originaire de l'est des Etats-Unis. II a été introduit en France dans un étang du Charolais à Chateauneuf (Saône et Loire) par Gensoul en 1910-1911 (GENSOUL, 1921), puis dans un étang proche de Lapalisse (Allier) en 1913, dans la commune de Sorbier (DODE, 1914, 1920). II était encore présent dans ce dernier en 1959 (SPILLMANN, 1961 ; ALLARDI et KEITH, 1991 ; KEITH 1998) et figure sur la liste des espèces représentées dans les cours d'eau français (arrêté du 17/12/85). Les captures les plus récentes ont été signalées en 1960 (à Jaligny, Allier) et en 1982 (à Givry en Argonne, Marne) par Mr Bourguignon puis par Mr Guidou en 1996 qui ont transmis à ces deux occasions des individus au Muséum National d'Histoire Naturelle.

Les premières observations d'umbres pygmées en Argonne datent des années 1970. L'acclimatation de l'espèce sur quelques sites en Belgique est certainement à 
l'origine de l'introduction dans la Marne. En effet, à cette période, la majorité des étangs Argonnais sont exploités par des pisciculteurs belges qui ont disséminé les umbres sur plusieurs étangs (lors des mise en charge, les pêches, le transport, etc.), dont certains semblent avoir été colonisés avec succès.

Le premier site où l'espèce a été observée dans les années 80 est l'étang " du Bâtard ». Ce site, où l'umbre s'est particulièrement bien acclimaté, semble à l'origine de la colonisation de tout le secteur. Les exploitants d'étangs ont tenté de l'éradiquer par des assecs et des pêches.

L'umbre pygmée ou petit poisson-chien (Figures 1 et 2) est un petit poisson au corps allongé, peu comprimé latéralement, de section presque circulaire. Le pédoncule caudal, très épais, est à peine marqué. La nageoire dorsale, très en arrière, a le bord arrondi. La tête porte un œil bien développé et une bouche petite avec de fines dents. L'insertion des nageoires pelviennes est nettement en avant de la dorsale. La nageoire caudale est unilobée et convexe. La dorsale est placée très en arrière, presque à l'aplomb de l'anale. Les écailles, grandes et arrondies recouvrent également le dessus de la tête, les joues et les opercules. Le dos est vert-brun, les flancs sont plus clairs avec des bandes longitudinales claires. Celle qui prend naissance près du bord supérieur de l'opercule est deux fois plus large que les autres. L'attache de la nageoire caudale au pédoncule caudal est soulignée par un liséré brun foncé à noir. La mâchoire inférieure est généralement noire. La taille varie de 4 à $10 \mathrm{~cm}$.

La dorsale a 14-15 rayons, l'anale 11 , les pectorales $15-16$, les pelviennes 6 , la caudale 18-20. La formulaire scalaire est : 6-7/32-37/9-11 (SPILLMANN, 1961 ; KEITH et ALLARDI, 2001).

L'umbre pygmée vit dans les marais, les mares peu profondes et les eaux lentes à fond bourbeux. II possède une grande résistance au manque d'oxygène et à la pollution. Il fraie de mars à avril. Les œufs sont pondus parmi la végétation des rives. L'incubation dure 5 à 10 jours. La maturité sexuelle survient la $2^{\text {ème }}$ année. II se nourrit de larves d'insectes, de petits mollusques et de crustacés planctoniques (Cyclops, daphnies) (KEITH et ALLARDI, 2001).

En novembre 2000, l'un d'entre nous (F.G.) décide d'approfondir les faibles connaissances disponibles sur la présence de l'umbre pygmée en Argonne (Marne), de prospecter des sites potentiels et d'interroger les propriétaires d'étangs susceptibles d'avoir connaissance de la présence de l'espèce. Monsieur Bourguignon, un pisciculteur, indiqua que l'espèce avait proliféré sur l'étang " le Bâtard ", près de Givry en Argonne, dans les années 1980, avec une biomasse de plus de $100 \mathrm{~kg}$ pour ce plan d'eau d'une superficie de 5 hectares. L'éradication de l'umbre avait été réalisée car l'espèce ne présentait aucun intérêt économique. L'umbre avait néanmoins subsisté grâce à la succession d'étangs vers l'aval : Braux-forêt, Fourchu et Grand Ru qui auraient été colonisés par les fossés de vidange lors des pêches annuelles.

Ces sites potentiels ont donc été prospectés pour essayer de localiser l'espèce. Un site, le ruisseau de Braux-Forêt, à l'aval de l'étang du même nom vidangé récemment, a permis la récolte d'umbres pygmées. A la suite de la vidange de l'étang de Braux-forêt, propriété de Monsieur Leblanc, une pêche électrique fut effectuée en aval de la pêcherie le 29 novembre 2000, afin d'essayer de récolter quelques individus. Lors du sondage, avec le martin-pêcheur, furent capturés des brochets, des perches, des gardons, des tanches, des ables et des umbres pygmées (595 individus dont les tailles variaient de 41 à 99 millimètres).

Ces poissons étaient concentrés dans la pêcherie de l'étang de Braux-Forêt et survivaient dans des conditions difficiles (peu d'eau, quantité de vase importante). 


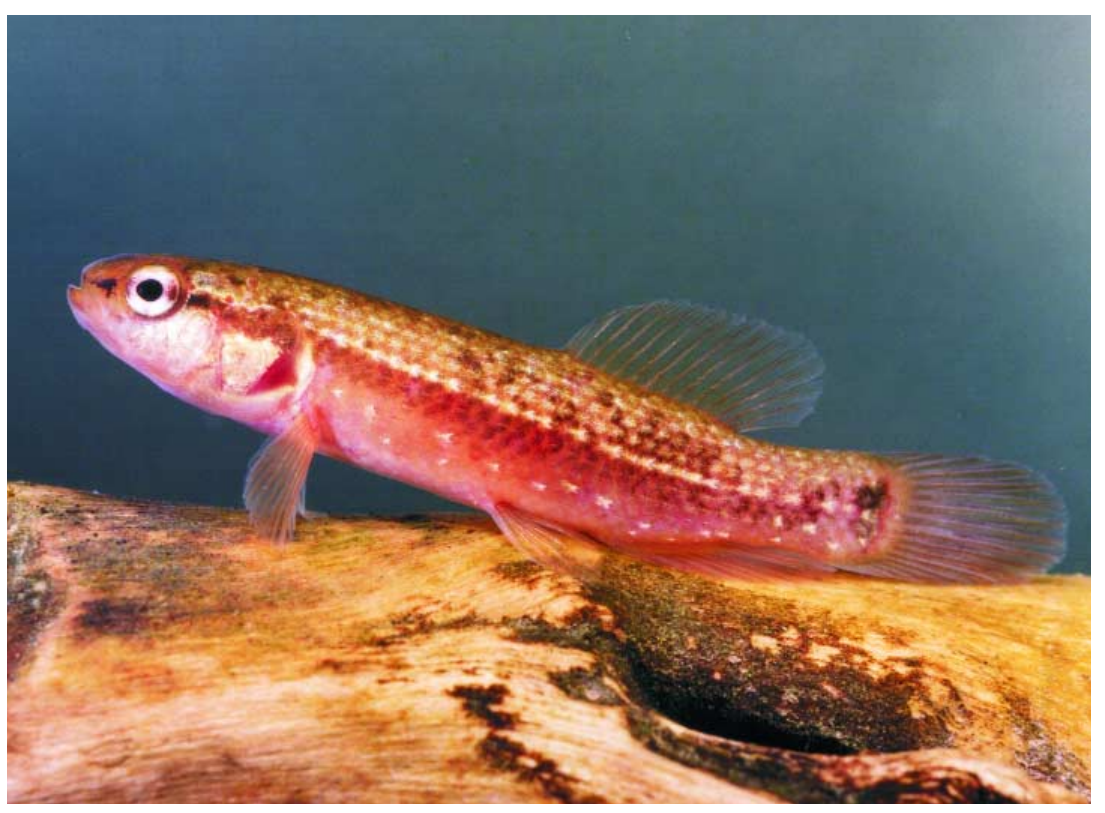

Figure 1

Vue générale de l'umbre pygmée (Photo E. Vigneux).

\section{Figure 1}

General view of Eastern Mudminnow (Photo E. Vigneux).

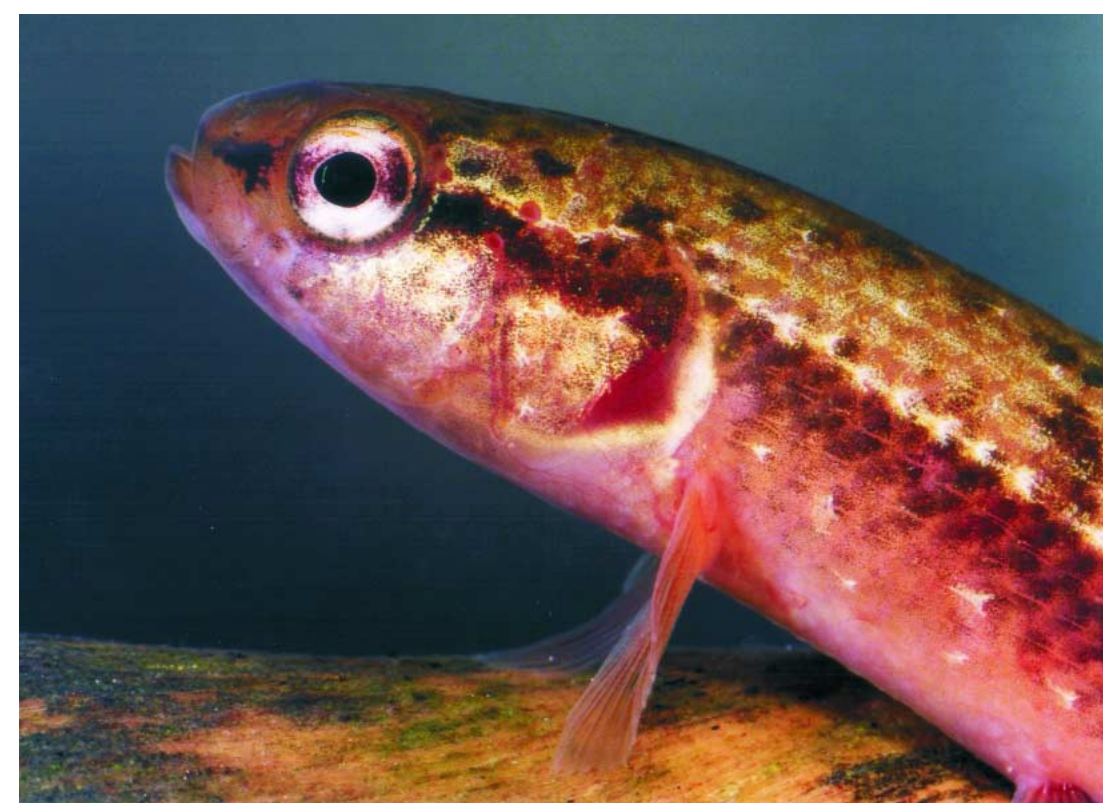

\section{Figure 2}

Gros plan de la partie antérieure (Photo E. Vigneux).

\section{Figure 2}

Anterior part of Eastern Mudminnow (Photo E. Vigneux). 
Bien que l'espèce soit toujours présente dans le département de la Marne, sa répartition est faible. Sa discrétion contribue à sa survie : elle peut passer inaperçue des années durant dans un plan d'eau, même pêché annuellement, si une attention toute particulière n'est pas observée lors du tri.

En collaboration avec l'Office National des Forêts (ONF), des exemplaires d'umbres capturés lors de la pêcherie de l'étang de Braux-Forêt ont pour l'instant été conservés dans un étang argonnais, l'étang Maujean, appartenant à l'ONF. La brigade du CSP, toujours en collaboration avec l'ONF, effectuera un suivi annuel des populations afin d'obtenir des renseignements sur la biologie de l'espèce: capacité d'acclimatation, reproduction, régime alimentaire, croissance, pathologie, etc. L'étang Maujean, qui a une superficie de 0,5 hectare, présentera également un aspect pédagogique de part les aménagements qui vont être réalisés.

\section{REMERCIEMENTS}

J.P. Raulin, J.C. Antoine et E. Vigneux du CSP ; J. Fouraux et W. Laguerre de I'ONF ; Messieurs Thillois, des Charmontois, Bourguignon et Leblanc.

\section{BIBLIOGRAPHIE}

ALLARDI J., KEITH P. (coord.), 1991. Atlas préliminaire des poissons d'eau douce de France. Coll. Patrimoines Naturels, vol. 4. SFF/MNHN, CSP, Cemagref, Min. Env., Paris, $234 \mathrm{p}$.

DODE L., 1914. Essai d'acclimatation de poissons exotiques en étang. Bull. Soc. cent. Aquic. Pêche, 26, 17-24.

DODE L., 1920. Naturalisation en étang de deux espèces de poissons des Etats-Unis (Umbra pygmaea De Kay et Centrarchus macropterus Lac.). Bull. Soc. cent. Aquic. Pêche, 27, 113-114.

GENSOUL J., 1921. De l'acclimatation de quelques poissons en eau libre. Bull. Soc. cent. Aquic. Pêche, 28, 47-49.

KEITH P., 1998. Evolution des peuplements ichtyologiques de France et stratégies de conservation. Thèse Université de Rennes I, $236 \mathrm{p}$.

KEITH P., ALLARDI J. (coord.), 2001. Atlas des poissons d'eau douce de France. Patrimoines naturels, 47, $387 \mathrm{p}$.

SPILLMANN C.J., 1961. Faune de France. Poissons d'eau douce. Editions Paul Lechevalier, Paris, $303 \mathrm{p}$. 